\title{
The soft Jacobson radical of a commutative ring
}

\author{
J. Ghosh, D. Mandal and T. K. Samanta
}

\begin{abstract}
In this paper, the notion of the soft Jacobson radical of a ring is defined. A relationship between the soft Jacobson radical of a ring and Jacobson semisimple ring is established. Some properties of this notion have been studied under homomorphism.
\end{abstract}

Key Words: Jacobson radical, Jacobson semisimple ring, soft maximal intideal, soft Jacobson radical

Mathematics Subject Classification 2010: 05C25, 16N20, 06D72

\section{Introduction}

The idea of a soft set was introduced by D. Molodtsov 14 in 1999 as a parametrized mathematical tool which has many applications in medical sciences, engineering, economics, etc. Maji and Roy [12] presented a beautiful problem where soft sets are applied in decision making. Applications of soft sets in algebra have been studied rapidly in the last two decades.

Algebraic structure in soft set theory was introduced by Aktas and Cagman [2] in 2007. They defined a soft group as a parametrized family of subgroups of the given group. Extending the notion of a soft group, several algebraic structures like soft ring [1], soft ideal [6], soft vector space [15] etc., have been introduced. In 2012, Cagman et al. [5] defined group structure on a soft set in a new way using set inclusion relation. This concept has been named as soft int-group. Already many researchers have spent their times to extend the notion of soft int-group for further development of soft set theory in the same direction. As a result, the notions of soft int-ring [7], soft int-field, soft int-module [4], soft int-ideal [7] etc, have been established. The soft radical of a soft int-ideal is defined in [9].

This paper focuses on introducing the concept of the soft Jacobson radical of a ring. We define the soft Jacobson radical of a commutative ring $R$ with 
unity as the soft intersection of all soft maximal int-ideals of $R$. A connection between the soft Jacobson radical of a ring and Jacobson semisimple ring has been studied. It is established that the homomorphic image of the soft Jacobson radical of a ring $R$ is equal to the soft Jacobson radical of a homomorphic image of $R$ under certain condition. If $f$ is an epimorphism from a ring $R$ to a ring $R^{\prime}$, then we prove that the homomorphic pre-image of the soft Jacobson radical of $R^{\prime}$ is equal to the soft Jacobson radical of $R$ under a suitable condition.

\section{Preliminaries}

We include some basic definitions and results of soft set theory which will be useful in the next section. Throughout this paper, unless otherwise is stated, $U$ is the initial universe, $E$ is the set of parameters, $P(U)$ is the power set of $U$, and $A \subseteq E$.

Let $F$ be a mapping given by $F: A \rightarrow P(A)$. A pair $(F, A)$ is called a soft set of $A$ over $U$. When no confusions regarding the parameter set $A$ and the universal set $U$ arise, the soft set $(F, A)$ is simply denoted by $F$. The collection of all soft sets with parameter set $A$ over $U$ will be denoted by $S(A, U)$.

Let $F, G \in S(A, U)$. If $F(t) \subseteq G(t)$ for all $t \in A$, then $F$ is called a soft subset of $G$ and is denoted by $F \widetilde{\subseteq} G$. Here $G$ is called a soft superset of $F$ and it is denoted by $G \supseteq \widetilde{\supseteq} F$. We write $F=G$, if $F(t)=G(t)$ for all $t \in A$.

Let $F \in S(A, U)$ and $K \subseteq U$, the set $F_{K}=\{x \in A: F(x) \supseteq K\}$ is called $K$-inclusion subset of the soft set $F$.

The soft intersection $F \widetilde{\cap} G$ of two soft sets $F$ and $G$ is defined by

$$
(F \widetilde{\cap} G)(t)=F(t) \cap G(t), \quad t \in A .
$$

It is not difficult to see that the following statement holds true.

Proposition 1 Let $F, G \in S(A, U)$ and $K \subseteq U$. Then $(F \widetilde{\cap} G)_{K}=F_{K} \cap G_{K}$.

Let $A$ and $A^{\prime}$ be some parameter sets and let $f: A \rightarrow A^{\prime}$ be any mapping. The soft image $f(F)$ of $F$ under $f$ is defined by

$$
f(F)(y)= \begin{cases}\bigcup_{x \in f^{-1}(y)} F(x), & \text { if } f^{-1}(y) \neq \emptyset \\ \emptyset, & \text { otherwise }\end{cases}
$$

for all $y \in A^{\prime}$.

Let $A, A^{\prime} \subseteq E, G \in S\left(A^{\prime}, U\right)$ and let $f: A \rightarrow A^{\prime}$ be an onto mapping. The soft preimage of $G$ under $f$ is a soft set $f^{-1}(G) \in S(A, U)$ such that $f^{-1}(G)(s)=G(f(s))$ for all $s \in A$. 
A soft set $F \in S(A, U)$ is called an $f$-invariant soft set if from $f(x)=$ $f(y)$ it follows that $F(x)=F(y), x, y \in A$.

Let $R$ be a ring. A soft set $F \in S(R, U)$ is called a soft int-ring of $R$ if (i) $F(x-y) \supseteq F(x) \cap F(y)$ and

(ii) $F(x y) \supseteq F(x) \cap F(y)$ for all $x, y \in R$.

A soft set $F \in S(R, U)$ is called a soft int-ideal of $R$ if

(i) $F(x-y) \supseteq F(x) \cap F(y)$ and

(ii) $F(x y) \supseteq F(x) \cup F(y)$ for all $x, y \in R$.

Proposition 2 [7] Let $F$ be a soft int-ring or soft int-ideal of a ring $R$. Then $F(0) \supseteq F(r)$ for all $r \in R$, where 0 is the zero element of $R$.

A soft int-ideal $F$ of a commutative ring $R$ is called a soft maximal int-ideal if $F_{L}$ is a maximal ideal of $R$ for $L=F(0)$.

Let $F$ be a soft int-ideal of a ring $R$ and $a \in R$. The soft set $F_{a}$ of $R$, defined by $F_{a}(r)=F(r-a), r \in R$, is called the soft coset of $F$ in $R$ determined by $a \in R$.

We denote the set of all soft cosets of a soft int-ideal $F$ of a ring $R$ by $R \curlywedge F$.

Theorem 1 [9] Let $F$ be a soft int-ideal of a ring $R$. Then $R$ ? $F$ forms a ring with respect to binary compositions $F_{a}+F_{b}=F_{a+b}$ and $F_{a} F_{b}=F_{a b}$ for all $a, b \in R$. Here $F_{0}$ is the zero element of $R 2 F$. If $R$ is a ring with unity 1 , then $F_{1}$ is the unity element in $R$ ? $F$.

Proposition 3 [9] Let $F$ be a soft int-ideal of a ring $R$. Then $F_{a}=F_{0}$ if and only if $F(a)=F(0)$, where $a \in R$.

Theorem 2 [10] Let $f: R \rightarrow R^{\prime}$ be an epimorphism, where $R, R^{\prime}$ are two commutative rings with unity. If $F$ is an $f$-invariant soft maximal int-ideal of $R$, then $f(F)$ is a soft maximal int-ideal of $R^{\prime}$.

Theorem 3 [10] Let $f: R \rightarrow R^{\prime}$ be a homomorphism, where $R, R^{\prime}$ are two commutative rings with unity. If $F^{\prime}$ is a soft maximal int-ideal of $R^{\prime}$, then $f^{-1}\left(F^{\prime}\right)$ is an $f$-invariant soft maximal int-ideal of $R$.

The following statement is easy to be verified.

Proposition 4 Let $A, A^{\prime} \subseteq E$ and $f: A \rightarrow A^{\prime}$ be any onto mapping. If $G \in S\left(A^{\prime}, U\right)$, then $f\left(f^{-1}(G)\right)=G$.

Proposition 5 [5] Let $A, A^{\prime} \subseteq E$ and $f: A \rightarrow A^{\prime}$ be any mapping. Let $F_{i} \in S\left(A^{\prime}, U\right)$ for all $i \in \Delta$, where $\Delta$ is the non-empty index set. Then $f^{-1}\left(\widetilde{\bigcap}_{i \in \Delta} F_{i}\right)=\bigcap_{i \in \Delta} f^{-1}\left(F_{i}\right)$. 
Proposition 6 [9] Let $f$ be any mapping from a set $A$ to a set $A^{\prime}$. If $F$ is an $f$-invariant soft set of $A$, then $f^{-1}(f(F))=F$.

A ring $R$ is called Jacobson semisimple ring if the Jacobson radical $J R(R)=\{0\}$.

Theorem 4 [13] The quotient ring $R / J R(R)$ is a Jacobson semisimple ring.

\section{Soft Jacobson Radical of a Ring}

Throughout this section, $R$ is a commutative ring with unity 1 . In ring theory [13], the Jacobson radical $J R(R)$ of the $\operatorname{ring} R$ is defined as the intersection of all maximal ideals of $R$. Here, we introduce the following notion.

The soft Jacobson radical $S J R(R)$ of the ring $R$ is defined by

$$
\operatorname{SJR}(R)=\widetilde{\bigcap}\{F: F \text { is a soft maximal int-ideal of } R\} .
$$

Theorem 5 [13] Let $y \in R$. Then $y \in J R(R)$ if and only if $1-x y$ is a unit in $R$ for all $x \in R$.

Theorem 6 Let $y \in R$ and $F=S J R(R)$. Then $y \in F_{K}$ with $K=F(0)$ if and only if $1-x y$ is a unit in $R$ for all $x \in R$.

Proof. Let $y \in F_{K}$ for $K=F(0)$. Suppose $1-x y$ is not a unit in $R$ for some $x \in R$. Then, by the crisp concept, there exists a maximal ideal $I$ of $R$ such that $1-x y \in I$. Define a soft int-ideal $H$ of $R$ over $U$ by

$$
H(r)= \begin{cases}N, & \text { if } r \in I, \\ L, & \text { if } r \in R-I,\end{cases}
$$

where $L \subset N \subseteq U, L \subset K$. Hence, $H$ is a soft maximal int-ideal of $R$. Now, $y \in F_{K}$ implies $F(y) \supseteq K$. Since $H$ is a soft maximal int-ideal of $R$ and $F=S J R(R)$, we get $H(y) \supseteq F(y)$. Thus, $H(y) \supseteq K \supset L$. This implies $H(y)=N$, and hence, $y \in I$. Then $x y \in I$. Therefore, $1=1-x y+x y \in I$. This implies that $I$ is not a maximal ideal of $R$, which is a contradiction. Therefore, $1-x y$ is a unit in $R$ for all $x \in R$.

Conversely, assume that $1-x y$ is a unit in $R$ for all $x \in R$. Then by Theorem 5, $y \in J R(R)$. Hence, $y \in M$ for any maximal ideal $M$ of $R$. Now, let $F=\bigcap_{i} F_{i}$, where $F_{i}$ is a soft maximal int-ideal of $R$ for $1 \leq i \leq n$, $i, n \in \mathbb{N}$. Since $\left(F_{i}\right)_{L_{i}}$ (where $\left.L_{i}=F_{i}(0)\right)$ is a maximal ideal of $R, y \in\left(F_{i}\right)_{L_{i}}$ for $1 \leq i \leq n$. Again, $K=F(0)=\bigcap_{i} F_{i}(0)$. Then $L_{i}=F_{i}(0) \supseteq K$ for $1 \leq i \leq n, n \in \mathbb{N}$. Therefore, $y \in\left(F_{i}\right)_{K}$ for all $i$. By Proposition 1 , $\left(\widetilde{\bigcap}_{i} F_{i}\right)_{K}=\bigcap_{i}\left(F_{i}\right)_{K}$. Hence, $y \in\left(\widetilde{\bigcap}_{i} F_{i}\right)_{K}=F_{K}$. 
Theorem 7 Let $F=S J R(R)$. Then $r \in R$ is a unit in $R$ if and only if $F_{r}$ is a unit in $R$ ? $F$.

Proof. Let $r \in R$ be a unit in $R$. Then there exists $s \in R$ such that $r s=1$. Hence, $F_{r s}=F_{1}$, where $F_{1}$ is the unity element in $R \imath F$ (by Theorem 1). This implies $F_{r} F_{s}=F_{1}$. Thus, $F_{r}$ is a unit in $R \imath F$.

Conversely, let $F_{r}$ be a unit in $R$ ? $F$ for $r \in R$. Then there exists $F_{s} \in R$ 乙 F such that $F_{r} F_{s}=F_{1}$. This implies $F_{r s}=F_{1}$, and therefore, $F(x-r s)=F(x-1)$ for all $x \in R$. Particularly, $F(1-r s)=F(0)$.

Let $I$ be any maximal ideal of $R$. Define a soft int-ideal $H$ of $R$ by

$$
H(a)= \begin{cases}F(0), & \text { if } a \in I, \\ L, & \text { if } a \in R-I,\end{cases}
$$

where $L \subset F(0)$. Then $H$ is a soft maximal int-ideal of $R$ and $H(0)=F(0)$. Since $F$ is the soft Jacobson radical of $R, F(0)=F(1-r s) \subseteq H(1-r s)$. This implies $H(1-r s)=H(0)$, and hence, $1-r s \in I$. Thus, $1-r s \in J R(R)$, since $I$ is any maximal ideal of $R$. Then by Theorem 5 , rs $=1-(1-r s)$ is a unit in $R$. Therefore, $r$ is a unit in $R$.

Theorem 8 Let $F=S J R(R)$. Then the ring $R \nmid F$ is a Jacobson semisimple ring.

Proof. To prove that the ring $R \curlywedge F$ is a Jacobson semisimple ring, we have to prove $J R(R \succ F)=\left\{F_{0}\right\}$. Let $F_{r} \in J R(R \succ F)$. Then by Theorem 5 , $F_{1}-F_{x} F_{r}$ is a unit in $R \imath F$ for all $F_{x} \in R \imath F$. There exists $F_{y} \in R \imath F$ such that $\left(F_{1}-F_{x} F_{r}\right) F_{y}=F_{1}$. By Theorem 1, $F_{(1-x r) y}=F_{1}$. Hence, $F_{(1-x r) y}(a)=F_{1}(a)$ for all $a \in R$. Then $F(a-y+x r y)=F(a-1), a \in R$. Therefore,

$$
F(1-y+x r y)=F(0) .
$$

Let $I$ be any maximal ideal of $R$. We define a soft int-ideal $H$ of $R$ by

$$
H(a)= \begin{cases}F(0), & \text { if } a \in I, \\ L, & \text { if } a \in R-I,\end{cases}
$$

where $L \subset F(0)$. Then $H$ is a soft maximal int-ideal of $R$ and $H(0)=F(0)$. Since $F$ is the soft Jacobson radical of $R$, Equation (1) implies

$$
H(1-y+x r y) \supseteq F(1-y+x r y)=F(0)=H(0) .
$$

By Proposition 2, $H(1-y+x r y)=H(0)$. Therefore, $1-y+x r y \in I$ for any maximal ideal $I$ of $R$. This implies $1-y+x r y \in J R(R)$. Then by Theorem 5, $1-(1-y+x r y)=y-x r y$ is a unit in $R$. This implies that $1-x r$ is a unit in $R$, and hence, $r \in J R(R)$. 
Suppose $\left\{G_{i}: i \in \Gamma\right\}$ is a collection of all soft maximal int-ideals of $R$, where $\Gamma$ is the index set. Then $\left(G_{i}\right)_{L_{i}}$ is the maximal ideal of $R$, where $L_{i}=G_{i}(0)$. From $r \in J R(R)$ it follows that $r \in\left(G_{i}\right)_{L_{i}}$. Hence, $G_{i}(r)=$ $L_{i}=G_{i}(0)$, and therefore,

$$
F(r)=\bigcap_{i \in \Gamma} G_{i}(r)=\bigcap_{i \in \Gamma} G_{i}(0)=F(0) .
$$

By Proposition 3, we have $F_{r}=F_{0}$. Thus, $J R(R \succ F)=\left\{F_{0}\right\}$. Therefore, $R$ 乙 $F$ is a Jacobson semisimple ring.

Proposition 7 Let $A, A^{\prime} \subseteq E$ and $f: A \rightarrow A^{\prime}$ be any onto mapping. Let $F_{1}, F_{2} \in S(A, U)$. If $F_{1}, F_{2}$ are both $f$-invariant, then $f\left(F_{1} \widetilde{\cap} F_{2}\right)=$ $f\left(F_{1}\right) \widetilde{\cap} f\left(F_{2}\right)$.

Proof. Since $F_{1}, F_{2}$ are both $f$-invariant soft sets, from $f(x)=f(y)$ it follows that $F_{1}(x)=F_{1}(y)$ and $F_{2}(x)=F_{2}(y)$, where $x, y \in A$. Hence, $\left(F_{1} \widetilde{\cap} F_{2}\right)(x)=F_{1}(x) \cap F_{2}(x)=F_{1}(y) \cap F_{2}(y)=\left(F_{1} \widetilde{\cap} F_{2}\right)(y)$. Therefore, $F_{1} \widetilde{\cap} F_{2}$ is also $f$-invariant soft set.

Now, for any $y \in A$, using the fact that $F_{1} \widetilde{\cap} F_{2}$ is $f$-invariant, we have

$$
\begin{aligned}
f\left(F_{1} \widetilde{\cap} F_{2}\right)(y) & =\underset{z \in f^{-1}(y)}{\cup}\left(F_{1} \widetilde{\cap} F_{2}\right)(z) \\
& =\left(F_{1} \widetilde{\cap} F_{2}\right)(y) \\
& =F_{1}(y) \cap F_{2}(y) .
\end{aligned}
$$

Since $F_{1}$ and $F_{2}$ are $f$-invariant, further we can write

$$
\begin{aligned}
& {\left[f\left(F_{1}\right) \tilde{\cap} f\left(F_{2}\right)\right](y)} \\
& =f\left(F_{1}\right)(y) \cap f\left(F_{2}\right)(y) \\
& =\left[\bigcup_{s \in f^{-1}(y)} F_{1}(s)\right] \cap\left[\bigcup_{t \in f^{-1}(y)} F_{2}(t)\right] \\
& =F_{1}(y) \cap F_{2}(y) .
\end{aligned}
$$

Therefore, $f\left(F_{1} \widetilde{\cap} F_{2}\right)=f\left(F_{1}\right) \widetilde{\cap} f\left(F_{2}\right)$.

The following proposition is a generalization of Proposition 7 for an arbitrary soft intersection.

Proposition 8 Let $A, A^{\prime} \subseteq E$ and $f: A \rightarrow A^{\prime}$ be any onto mapping. Let $F_{i} \in S(A, U)$ be $f$-invariant for all $i \in \Gamma$, where $\Gamma$ is an arbitrary non-empty index set. Then $f\left(\bigcap_{i \in \Gamma} F_{i}\right)=\bigcap_{i \in \Gamma} f\left(F_{i}\right)$. 
Theorem 9 Let $f: R \rightarrow R^{\prime}$ be a homomorphism, where $R, R^{\prime}$ are two commutative rings with unity. If each soft maximal int-ideal of $R$ is $f$ invariant, then $f(S J R(R))=S J R(f(R))$.

Proof. Let $\left\{F_{i}: i \in \Gamma\right\}$ be the complete collection of soft maximal int-ideals of $R$, where $\Gamma$ is an arbitrary non-empty index set. Then by the definition of soft Jacobson radical, we have $S J R(R)=\widetilde{\bigcap}_{i \in \Gamma} F_{i}$. Let $F_{i}$ be $f$-invariant for all $i \in \Gamma$. Then by Proposition 8 , we have

$$
f(S J R(R))=f\left(\widetilde{\bigcap_{i \in \Gamma}} F_{i}\right)=\widetilde{\bigcap_{i \in \Gamma}} f\left(F_{i}\right) .
$$

Since each $F_{i}(i \in \Gamma)$ is an $f$-invariant soft maximal int-ideal of $R$, by Theorem 2, we have $f\left(F_{i}\right)$ is a soft maximal int-ideal of $f(R)$ for all $i \in \Gamma$.

Suppose $G$ is a soft maximal int-ideal of $f(R)$. Then by Theorem 3 , $f^{-1}(G)$ is an $f$-invariant soft maximal int-ideal of $R$. Hence, by Proposition 4. $f\left(f^{-1}(G)\right)=G$. Thus, $\left\{f\left(F_{i}\right): i \in \Gamma\right\}$ is the complete collection of soft maximal int-ideals of $f(R)$. Hence, $\bigcap_{i \in \Gamma} f\left(F_{i}\right)=S J R(f(R))$. Therefore, $f(S J R(R))=S J R(f(R))$.

Theorem 10 Let $f: R \rightarrow R^{\prime}$ be an epimorphism, where $R, R^{\prime}$ are two commutative rings with unity. Then $f^{-1}\left(S J R\left(R^{\prime}\right)\right) \supseteq$ SJ $R(R)$. Moreover, if each soft maximal int-ideal of $R$ is $f$-invariant, then $f^{-1}\left(S J R\left(R^{\prime}\right)\right)=$ $\operatorname{SJR}(R)$.

Proof. Let $\left\{G_{j}: j \in \Delta\right\}$ be the complete collection of all soft maximal int-ideals of $R^{\prime}$. Hence, by the definition of soft Jacobson radical, we have $\operatorname{SJR}\left(R^{\prime}\right)=\widetilde{\bigcap_{j \in \Delta}} G_{j}$. By Proposition 5 .

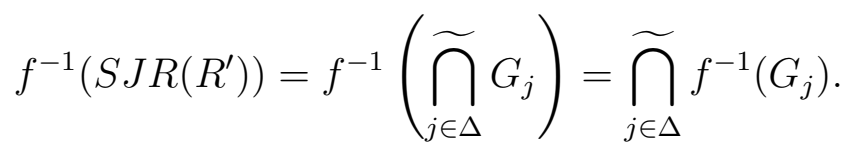

By Theorem 3, $f^{-1}\left(G_{j}\right)$ is an $f$-invariant soft maximal int-ideal of $R$ for all $j \in \Delta$. There may be some soft maximal int-ideal of $R$ that are not $f$-invariant. Hence,

$$
\widetilde{\bigcap_{j \in \Delta}} f^{-1}\left(G_{j}\right) \cong S J R(R) .
$$

Therefore, $f^{-1}\left(S J R\left(R^{\prime}\right)\right) \supseteq \widetilde{\supseteq} S R(R)$.

Now, we assume that each soft maximal int-ideal of $R$ is $f$-invariant. Let $F$ be a soft maximal int-ideal of $R$. Then by Theorem $2, f(F)$ is a soft maximal int-ideal $R^{\prime}$ and by the Proposition $6, f^{-1}(f(F))=F$. Hence, 
for each soft maximal int-ideal $F$ of $R$, there exist soft maximal int-ideal $f(F)$ of $R^{\prime}$ such that $f^{-1}(f(F))=F$. Thus, from Equation (2), we have $\widetilde{\bigcap}_{j \in \Delta} f^{-1}\left(G_{j}\right)=S J R(R)$. Therefore, $f^{-1}\left(S J R\left(R^{\prime}\right)\right)=S J R(R)$.

\section{Acknowledgments}

The authors are grateful to the reviewers and the editors of this journal for their comments and valuable suggestions to modify the paper.

\section{References}

[1] U. Acar, F. Koyuncu, and B. Tanay, Soft sets and soft rings, Comput. Math. Appl., 59 (2010), no. 11, pp. 3458-3463. https://doi.org/10.1016/j.camwa.2010.03.034

[2] H. Aktas, and N. Cagman, Soft sets and soft groups, Inform. Sci., 177 (2007), pp. 2726-2735.

[3] M.I. Ali, F. Feng, X. Liu, W.K. Min, and M. Shabir, On some new operations in soft set theory, Comput. Math. Appl., 57 (2009), no. 9, pp. 1547-1553.

[4] A.O. Atagün, and A. Sezgin, Soft substructures of rings, fields and modules, Comput. Math. Appl., 61 (2011), no. 3, pp. 592-601. https://doi.org/10.1016/j.camwa.2010.12.005

[5] N. Cagman, F. Citak, and H. Aktas, Soft int-group and its applications to group theory, Neural Comput. Appl., 21 (2012), pp. 151-158.

[6] Y. Celik, C. Ekiz, and S. Yamak, A new view on soft rings, Hacettepe J. Math. Stat., 40 (2011), no. 2, pp. 273-286.

[7] F. Citak, and N. Cagman, Soft int-rings and its algebraic applications, J. Intell. Fuzzy Syst., 28 (2015), no. 3, pp. 1225-1233.

[8] F. Feng, Y.B. Jun, and X. Zhao, Soft semirings, Comput. Math. Appl., 56 (2008), pp. 2621-2628.

[9] J. Ghosh, D. Mandal, and T.K. Samanta, Soft semiprimary int-ideals of a ring, Analele Universitatii Oradea Fasc. Matematica, 25 (2018), no. 1 , pp. 141-151.

[10] J. Ghosh, D. Mandal, and T.K. Samanta, Soft maximal and irreducible int-ideals of a ring, New Math. Nat. Comput., 16 (2020), no. 1, pp. 37-52. 
[11] K. Kaygisiz, Homomorphism and isomorphism of soft int-groups, Afr. Mat., 29 (2018), pp. 641-654. https://doi.org/10.1007/s13370-0180566-4

[12] P.K. Maji and A.R. Roy, An application of soft sets in a decision making problem, Comput. Math. Appl., 44 (2002), pp. 1077-1083.

[13] D.S. Malik, J.M. Mordeson, and M. K. Sen, Fundamentals of Abstract Algebra, The McGraw-Hill Companies Inc., 1997.

[14] D. Molodtsov, Soft set theory-first results, Comput. Math. Appl., 37 (1999), pp. 19-31.

[15] A.S. Sezer, and A.O. Atagün, A new kind of vector space : Soft vector space, Southeast Asian Bull. Math., 40 (2016), pp. 753-770.

[16] A.S. Sezer, N. Çağman, A.O. Atagün, M. I. Ali, and E. Türkmen, Soft intersection semigroups, ideals and bi-ideals; a new application on semigroup theory I, Filomat, 29 (2015), no. 5, pp. 917-946. https://doi.org/10.2298/FIL1505917S

Jayanta Ghosh

Department of Pure Mathematics,

University of Calcutta,

35 Ballygunge Circular Road, Kolkata-700019, India.

ghoshjay_04@yahoo.com

Dhananjoy Mandal

Department of Pure Mathematics,

University of Calcutta,

35 Ballygunge Circular Road, Kolkata-700019, India.

dmandal.cu@gmail.com

Tapas Kumar Samanta

Department of Mathematics,

Uluberia College,

Uluberia, Howrah-711315, India.

mumpu_tapas5@yahoo.co.in

Please, cite to this paper as published in

Armen. J. Math., V. 13, N. 11(2021), pp. 19

https://doi.org/10.52737/18291163-2021.13.11-1-9 